\title{
Financial Tools for the Abatement of Traffic Congestion: A Dynamical Analysis
}

\author{
Angelo Antoci · Marcello Galeotti · Davide Radi
}

Received: 22 January 2011 / Accepted: 6 August 2011 / Published online: 30 August 2011

(C) Springer Science+Business Media, LLC. 2011

\begin{abstract}
In this article we propose a simple mechanism aimed at implementing and supporting environmental protection policies in urban areas based on innovative financial instruments issued by a policy maker (PM), which can be buyed by two categories of involved agents, city users (CU) and agencies providing the city services. According to this mechanism, virtuous service providers choosing to offer high quality services can obtain cost abatement. CU, reciprocally, have to pay for entering into the city, but can protect themselves against a city low quality of life by a self-insurance device. The interaction of these two categories of economic agents is modelled by a two-population evolutionary game, where the population of CU strategically interacts with that of service providers. From the analysis of the model it emerges that such a dynamics may lead to a welfare-improving attracting Nash equilibrium at which all CU choose to use environmental-friendly means of transportation and all service providers choose to offer high quality services. However, the basin of attraction of that equilibrium may have a rather complex morphology. In particular more attractors and/or limit cycles can be present. In such a context we indicate sufficient conditions making the virtuous equilibrium a global attractor for all trajectories starting at a mixed-strategy point.
\end{abstract}

\footnotetext{
A. Antoci

Dept. of Economics, Enterprise and Legislation, University of Sassari, via Torre Tonda 34, 07100 Sassari, SS, Italy

M. Galeotti

Dept. of Mathematics for Decisions, University of Florence, via Lombroso 6/17, 50134 Florence, FI, Italy

D. Radi $(\bowtie)$

"Lorenzo Mascheroni" Dept. of Mathematics, Statistics, Computing and Applications,

University of Bergamo, via dei Caniana, 2, 24127 Bergamo, BG, Italy

e-mail: davide.radi@unibg.it
} 
Keywords Replicator equations · Evolutionary dynamics ·

Environmental protection policies $\cdot$ Financial options

\section{Introduction}

Air pollution and the dangers for pedestrians and cyclists deriving from the widespread use of private cars in urban centres may incentive the use of private cars by city users (CU). The choice of using the car in these areas instead of going by bicycle or on foot has a self-enforcing nature: the higher the urban air pollution and traffic congestion levels, the higher the incentive to go by car to reduce one's exposure to these problems. An increase in the use of private cars further increases air pollution and the dangers of urban traffic in its turn, thus reinforcing the decision to go by car. As showed by the literature on environmental self-protection choices (see, e.g., Hueting 1980; Bonatti and Campiglio 2009; Antoci and Sodini 2009; Antoci et al. 2005, 2007a, 2010), this mechanism may lead the urban community towards suboptimal Nash equilibria characterized by an excessive use of cars, unbearable levels of air pollution and traffic congestion.

Therefore, such a context calls for the definition of a system of sustainable mobility based on road pricing schemes (see e.g. Gwilliam 2008), according to which the policy maker (PM) increases the costs to enter into urban areas by car (parking taxes, congestion charges and so on) and collects revenues which can be used to finance private or public firms providing services aimed at the abatement of the negative effects of urban traffic and at the improvement of the quality of life in urban agglomerates (e.g. the management of public transport networks, of cycle and pedestrian lanes, of small-scale urban green areas etc.). Those services can reduce the relative convenience of entering into urban areas by private cars and consequently can stop the undesirable self-enforcing process described above.

Road pricing instruments have been used to control traffic in several urban centres (e.g. Bergen, London, Milan, Singapore, Shanghai, Stockholm). The effectiveness of such policy instruments highly depends on the amount of the revenues raised via road pricing and on the quality of services provided to defend individuals from the effects due to traffic congestion. Service providers that furnish high quality services bear extra-costs and PMs have to give monetary incentives which reduce unitary costs. In this context, an increase in the proportion of individuals using private cars generates an increase in traffic congestion but at the same time leads to an increase in the raised funds, which can be used to incentive the provision of high quality services.

All the proposals set forth to reduce traffic congestion are based on fixed taxes that individuals have to pay when entering into urban areas by private cars. In our paper we propose a simple mechanism aimed at implementing and supporting environmental protection policies in urban areas based on innovative financial instruments issued by a PM, which can be bought by two categories of involved agents, CU and agencies providing the city services (SP), which can be public or private. In particular, we consider the case of a city whose citizens and visitors (the $\mathrm{CU}$ ) face the risk of a reduction in quality of life, caused by urban traffic, on one hand, and by a poor quality standard of urban services, on the other. 
According to the proposed mechanism, each CU has to choose (ex-ante) whether to use a private car (choice $(a)$ ) or to use a more environment-preserving transport mode-foots, cycles, buses, trolleybuses, trams (choice $(b)$ ). According to their choices the PM requires them to buy two different tickets, including cash-or-nothing call options, ${ }^{1}$ called, respectively, A (at a price $p_{a}$ ) and B (at a price $p_{b}<p_{a}$ ). The ticket prices, $p_{a}$ and $p_{b}$, are fixed by the PM. On the other hand, each SP has to choose (ex-ante) whether to improve (choice $(c)$ ) or not (choice $(d)$ ) the services it furnishes. Accordingly the PM requires them to subscribe two different contracts, similar to cash-or-nothing put options, called, respectively, C and D.

The tickets A and B imply a cost for the CU if the value of a properly defined index $Q$ of the city quality of life, measured by an independent agency at the end of any fixed period, is above a fixed threshold value $Q \geq Q^{*}$, but offer a reimbursement in the case $Q<Q^{*}$. When $Q<Q^{*}$, the CU owning the ticket A receive a reimbursement equal to $\alpha p_{a}$, while the $\mathrm{CU}$ owning the ticket $\mathrm{B}$ receive a reimbursement equal to $\beta p_{b}$, where $\alpha$ and $\beta$ are two parameters satisfying the condition $0<\alpha \leq \beta \leq 1(\alpha=\beta=1$ means that both amounts $p_{a}$ and $p_{b}$ are totally reimbursed). If $Q \geq Q^{*}$ the CU do not receive any refund.

On the side of the SP, those subscribing contract $\mathrm{C}$, i.e. improving the services they furnish, will bear a unitary extra-cost but, if the quality target $Q^{*}$ is achieved, will get a reward.

The contract $\mathrm{C}$ is very similar to the contract on which the "environmental policy bonds" regime, introduced by Horesh (2000a,b, 2002a,b) ${ }^{2}$, is based. Environmental policy bonds are auctioned by the Public Administration on the open market, but, unlike ordinary bonds, can be redeemed at the face value only if a specified environmental objective has been achieved. They do not bear any interest, and the yield investors can gain depends on the difference between the auctioned price and the face value in the case of redemption. Economic agents involved in the environmental objective, either polluters or not, once in possession of the bonds, have a strong interest to operate in such a way that the objective itself is quickly achieved, so to cash in the expected gains as soon as possible. Differently from the environmental policy bonds regime, the contract $\mathrm{C}$ considered in our model can be subscribed only by SP which intend to provide high quality services (the quality of services is assumed to be observable).

The tickets $\mathrm{A}$ and $\mathrm{B}$, bought by the $\mathrm{CU}$, can be regarded as the joint implementation of a fixed environmental tax and a potential refund. The prospective of a refund, in case $Q<Q^{*}$, makes these policy instruments more acceptable to public opinion; in fact, they can be considered as self-insurance products whose purchase can offer protection (or mitigation) from some environmental risk. By relying on the citizens aversion to environmental risks, these self-insurance instruments can be a partial alternative to new taxes or forms of public indebtedness.

\footnotetext{
1 A (call or put) cash-or-nothing option is a type of option whose payoff is either some fixed amount of cash or nothing at all (see, e.g., Hull 2005).

2 Even before Horesh, different types of financial instruments have been proposed to achieve social and environmental goals (e.g., see Perrings (1989), Costanza and Perrings (1990) and Torsello and Vercelli (1998)).
} 
According to the mechanism we propose, virtuous service providers choosing to offer high quality services can obtain cost abatement by subscribing the environmental contracts $\mathrm{C}$. CU have to pay to enter into the city, but can protect themselves against a low city quality of life by a self-insurance device. The PM can achieve the goal of improving the city quality of life at a low cost, since the costs born by CU compensate, at least partially, the financial aids to virtuous services providers and so do not imply any worsening of the public budget. In such a context, an increase in the number of CU choosing to enter into the city by private cars has a negative effect on the value of the quality index $Q$; however, since they have to pay a higher ticket $\left(p_{a}>p_{b}\right)$, they contribute to increase the funds used to incentive virtuous behavior by service providers, which, in turn, contributes to increase the value of $Q$. Therefore, as a consequence of the mechanism described above, a strong interdependency between $\mathrm{CU}$ and services providers' behavior occurs. The aim of this paper is to study the dynamics that may arise in such a context. To this purpose, the choice processes of CU and service providers are modelled via a two-population evolutionary game, where the population of CU strategically interacts with that of service providers. Specifically these processes are modelled by the so-called replicator dynamics (e.g., see Weibull 1995), according to which a given choice spreads among the population as long as its expected payoff is greater than the average one. As it emerges from the model, such a dynamics may lead to a welfare-improving attracting Nash equilibrium, in which all CU choose to use environmentally-friendly means of transportation and all service providers choose to offer high quality services. The basin of attraction of this equilibrium expands as the reimbursement due to the visitors increases.

The model presented in this paper is a generalization of the model analyzed in Antoci et al. (2009a). In Antoci et al. (2007b, 2009b,c) as well as in Bischi and Radi (2011) a similar fund rising mechanism has been analyzed in a context in which firms have to decide about the adoption of an environment preserving technology.

The structure of the remainder of the paper is as follows. Section 2 develops the game theoretic model; in Sects. 3 and 4 the model is analyzed; Sect. 5 concludes.

\section{The Model}

We assume that, at each time $t, \mathrm{CU}$ and agencies providing the city services (SP) play a one shot population game (i.e. all CU and all SP play the game simultaneously). Each $\mathrm{CU}$ has to choose (ex-ante) whether to use a private car (choice $(a)$ ) or not (choice (b)). The PM requires them to buy two different tickets, including cash-or-nothing call options, called A (at a price $p_{a}$ ) and B (at a price $p_{b}$ ), according to their choices. Each SP has to opt (ex-ante) between choice $(c)$ and choice $(d)$ ), i.e. whether to improve or not the services it furnishes. The PM requires them to subscribe two different contracts, similar to cash-or-nothing put options, called C and D, according to their choices.

We assume the two populations to be constant over the time and normalize to 1 the number of both CU and SP. Let the variable $x(t)$ denote the proportion of CU adopting choice A at time $t(0 \leq x(t) \leq 1)$ and $1-x(t)$ the proportion of $\mathrm{CU}$ adopting choice B. Analogously, let $y(t)$ denote the proportion of SP choosing option $\mathrm{C}$ at the time $t(0 \leq y(t) \leq 1)$, and $1-y(t)$ the proportion of SP choosing option D.

The ticket prices, $p_{a}>p_{b}$, are fixed by the PM. 
The index $Q$ is a measure of the city quality of life, whose target is fixed by the PM at a sufficiently high value $Q^{*}$. We imagine that, at the end of any fixed period, an independent agency measures $Q$.

At the end of a period in which $Q<Q^{*}$, the $\mathrm{CU}$ owning the ticket A receive a reimbursement equal to $\alpha p_{a}$, while the $\mathrm{CU}$ owning the ticket $\mathrm{B}$ receive a reimbursement equal to $\beta p_{b}$, where $\alpha$ and $\beta$ are two parameters satisfying the condition $0<\alpha \leq \beta \leq 1\left(\alpha=\beta=1\right.$ means that both amounts, $p_{a}$ and $p_{b}$, are totally reimbursed). If $Q \geq Q^{*}$ the $\mathrm{CU}$ do not receive any refund (this means that the value of the option is zero). Hence the option values depend on the index $Q$ (or $-Q$ ), which is their underlying.

It is also reasonable to assume that each CUs payoff is affected not only by the price of the tickets and the amount of the reimbursements, but by the city quality of life, depending negatively on the use of private cars and positively on better urban services.

Consequently we assume the payoff of a CU buying ticket $\mathrm{A}$ to be given by:

- $\pi_{a}^{1}=\gamma_{1} y-\delta x-\rho p_{a}$ if $Q \geq Q^{*}$

- $\pi_{a}^{2}=\gamma_{2} y-\delta x-\rho(1-\alpha) p_{a}$ if $Q<Q^{*}$

while the payoff of a $\mathrm{CU}$ buying ticket $\mathrm{B}$ will be given by:

- $\pi_{b}^{1}=\varepsilon_{1} y-\eta x-\rho p_{b}$ if $Q \geq Q^{*}$

- $\pi_{b}^{2}=\varepsilon_{2} y-\eta x-\rho(1-\beta) p_{b}$ if $Q<Q^{*}$

All the parameters are positive. Moreover, $\gamma_{1}>\gamma_{2}, \varepsilon_{1}>\varepsilon_{2}, \varepsilon_{i}>\gamma_{i}, \varepsilon_{1}-\varepsilon_{2}>$ $\gamma_{1}-\gamma_{2}$, implying, in particular, that the citizens not using private cars derive more advantage from increases in service quality. All the citizen payoffs are negatively correlated to the number of citizens using private cars by the two parameters $\delta$ and $\eta$ (we assume $\eta \geq \delta$ ). Notice that both $p_{a}$ and $p_{b}$ are multiplied by $\rho$. This parameter can be thought as a measure of the citizen willingness to pay for urban services. For the sake of simplicity, we assume that all the citizens have the same willingness.

On the side of the SP, those adopting choice $(c)$, i.e. improving the services they furnish, will bear a unitary extra-cost $\theta, \theta>0$, but, if the quality target is achieved, will get a reward $\lambda+\mu x-v y$, where $\lambda-v>\theta, \mu, v>0$. The parameter $\theta$ represents the cost of increasing the level of services, while $\lambda$ is a fixed amount of money; $\mu>0$ means that the reward is positively related to the number of $\mathrm{CU}(x)$ who decide to use a private car. The reason is obvious: if we fix $p_{a}>p_{b}$, the more are the citizens buying ticket A, the larger are the financial resources available to pay the SP reward. On the other hand, an increase in $y$ implies that more SP will be entitled to the financial aid, thus reducing the reimbursement available to each one. Therefore, the payoff of a SP adopting choice $(c)$ is given by:

- $\pi_{c}^{1}=\lambda+\mu x-v y-\theta$ if $Q \geq Q^{*}$

- $\pi_{c}^{2}=-\theta$ if $Q<Q^{*}$

Without loss of generality, we can normalize to zero the payoff of a SP adopting choice $(d)$ (i.e. deciding not to provide high quality services).

Finally we assume:

$$
P\left(Q \geq Q^{*}\right)=\sigma(1-x)+(1-\sigma) y, \quad 0<\sigma<1
$$


where $P$ denotes a probability. This is equivalent to saying that, if all SP improve the services they provide and all CU renounce to private cars, then $Q$ will be almost surely above the threshold level $Q^{*}\left(P\left(Q \geq Q^{*}\right)=1\right)$. The parameter $\sigma$ represents the weight of $\mathrm{CU}$ renouncing to private cars on the probability that $Q \geq Q^{*}$.

Finally we obtain the expected payoffs:

- $E \pi_{a}=\left(\gamma_{1} y-\delta x-\rho p_{a}\right) P\left(Q \geq Q^{*}\right)+\left[\gamma_{2} y-\delta x-\rho(1-\alpha) p_{a}\right] P\left(Q<Q^{*}\right)$

- $E \pi_{b}=\left(\varepsilon_{1} y-\eta x-\rho p_{b}\right) P\left(Q \geq Q^{*}\right)+\left[\varepsilon_{2} y-\eta x-\rho(1-\beta) p_{b}\right] P\left(Q<Q^{*}\right)$

- $E \pi_{c}=(\lambda+\mu x-v y-\theta) P\left(Q \geq Q^{*}\right)-\theta P\left(Q<Q^{*}\right)$

- $E \pi_{d}=0$

The process of adopting strategies is modelled by the so called replicator dynamics (see, e.g., Weibull 1995), according to which the strategies whose expected payoffs are greater than the average payoff spread within the populations at the expense of the others. In our case:

$$
\begin{aligned}
& \dot{x}=x\left(E \pi_{a}-\overline{E \pi}_{C U}\right) \\
& \dot{y}=y\left(E \pi_{c}-\overline{E \pi}_{S P}\right)
\end{aligned}
$$

where

$$
\begin{aligned}
& \overline{E \pi}_{C U}=x \cdot E \pi_{a}+(1-x) \cdot E \pi_{b} \\
& \overline{E \pi}_{S P}=y \cdot E \pi_{c}+(1-y) \cdot E \pi_{d}
\end{aligned}
$$

are the average payoffs, respectively, of the two populations of CU and SP.

We assume that, in our context, replicator dynamics is generated by the following "expectation forming" mechanism. At the end of each period $t$ (whose length, in a continuous time framework, is reduced to zero), the values of $x$ and $y$ become common knowledge to the agents (e.g. one can imagine that these values are frequently reported and updated on the web-page of the Public Administration and on the local media). On the basis of such values, agents form their expectations about the relative performance of the available strategies in the next period (in other words, the current values of $x$ an $y$ are used as a proxy for the values of these variables in the close future).

The replicator system (1) in the square $[0,1]^{2}$ can be written as:

$$
\begin{aligned}
& \dot{x}=x(1-x)\left(E \pi_{a}-E \pi_{b}\right)=x(1-x) F(x, y) \\
& \dot{y}=y(1-y)\left(E \pi_{c}-E \pi_{d}\right)=y(1-y) G(x, y)
\end{aligned}
$$

\section{Setting}

$$
\begin{aligned}
\varphi & =\left(\varepsilon_{1}-\gamma_{1}\right)-\left(\varepsilon_{2}-\gamma_{2}\right) \\
\psi & =\varepsilon_{2}-\gamma_{2} \\
\zeta & =\eta-\delta \\
\tau & =\rho\left(\beta p_{b}-\alpha p_{a}\right) \\
\omega & =\rho\left[(1-\alpha) p_{a}-(1-\beta) p_{b}\right]
\end{aligned}
$$


we obtain:

$$
\begin{aligned}
& F(x, y)=(-\varphi y+\tau)[\sigma(1-x)+(1-\sigma) y]+\zeta x-\psi y-\omega \\
& G(x, y)=(\lambda+\mu x-v y)[\sigma(1-x)+(1-\sigma) y]-\theta
\end{aligned}
$$

where:

$$
\varphi, \psi>0, \zeta \geq 0, \omega>\max (0, \tau) ; \quad 0<\sigma<1 ; \lambda, \mu, \nu, \theta>0, \lambda-\nu>\theta
$$

\section{Fixed Points and Stability}

The analysis of fixed points of (2) and their stability is summarized by the following Propositions.

Proposition 1 The boundary fixed points are at least four (the vertices of $\left.[0,1]^{2}\right)$ and at most nine (the maximum can be attained). At least one and at most four of them are attractors: in particular, the vertex $(0,1)$ is always attracting and the four vertices are all attracting if and only if the boundary fixed points are eight.

Proof The conditions (3) imply, as it is easily checked, that the vertex $(0,1)$ is attracting. Moreover the hyperbola $F(x, y)=0$, having asymptotes $y=\frac{\sigma \tau-\zeta}{\sigma \varphi}$ and $-\sigma x+$ $(1-\sigma) y=c$, for a suitable $c$, has at most one intersection with each horizontal edge of the square $[0,1]^{2}$. On the other hand $G(x, y)=0$ has one intersection with $x=0$ at some point $(0, \bar{y})$ with $\bar{y}>1$. It follows that $G(x, y)=0$ has at most one intersection with the vertical edge $x=0$ of the square and at most two intersections with the vertical edge $x=1$. Hence the boundary fixed points are at most nine. Moreover, as in $(0,0)$ and $(0,1) F(x, y)<0$, the possible fixed points on the open horizontal edges cannot be attracting. Analogously, since $G(x, y)>0$ in $(0,1)$ and $G(x, y)<0$ in $(1,0)$, the possible fixed point on the open vertical edge $x=0$ and one of the possible fixed points on the open vertical edge $x=1$ cannot be attracting either. In fact, it follows that, when the boundary fixed points are eight, the four vertices are all attracting. In case of nine boundary fixed points, instead, the vertices $(0,0),(0,1)$ and $(1,0)$ are still attracting, while $(1,1)$ is a saddle and there exists a fourth attractor on the open edge $x=1$. In all the other cases the attractors are less than four.

Proposition 2 The minimum number of interior fixed points (i.e. belonging to $\left.(0,1)^{2}\right)$ is zero and the maximum (which can be attained) is three. At most one of them is an attractor and at most one of them is a saddle. When the interior fixed points are three, two of them are repellors and one is a saddle. Moreover, in this case, there exist trajectories starting (i.e. as $t \rightarrow-\infty)$ from the repellor with higher coordinates and tending (as $t \rightarrow+\infty$ ) to the boundary of the square

Proof We omit the rather lengthy but straightforward proof, referring the interested reader to Antoci et al. (2011).

According to Propositions 1 and 2, the desirable equilibrium $(x, y)=(0,1)$, where all $\mathrm{CU}$ choose an environment-preserving transport mode and all services agencies 


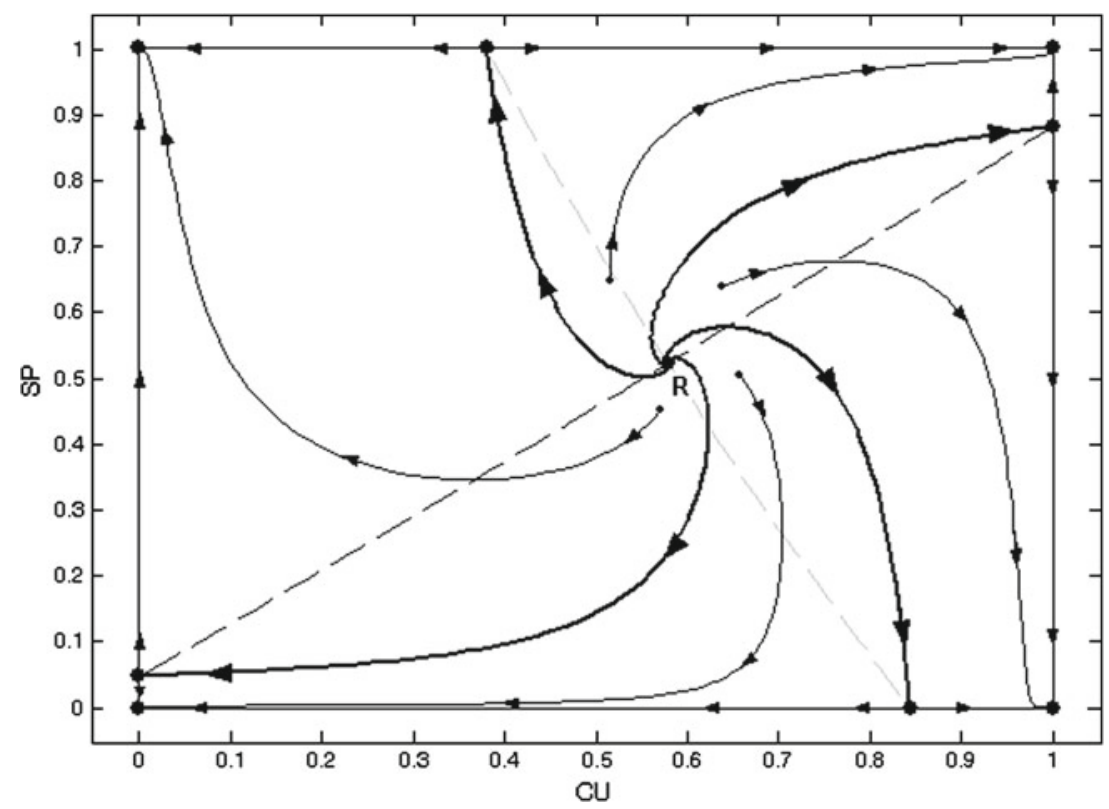

Fig. 1 All the four vertices of the square are attractors. Parameters: $\lambda=460, \mu=120, v=40, \psi=$ $48, \varphi=25, \omega=320, \theta=240, \tau=315, \sigma=0.5, \zeta=350$

provide high quality services, is always a local attractor under evolutionary dynamics. However, other fixed points can be simultaneously locally attractive; in such a context, the equilibrium selection depends on the initial distributions $(x(0), y(0))$ of strategies in the two interacting populations. In fact the morphology of the basin of attraction of $(0,1)$ may be, in this case, rather complex: for example, when a repelling limit cycle exists (as shown in the next section), the separatrices of different basins can wind around the cycle.

Figure 1 illustrates the case when the boundary fixed points are eight and thus all the four vertices are attractors. Figure 2 shows an example where three interior fixed points exist.

\section{Limit Cycles and Bifurcations}

The main result of this section is the proof that system (2), defined in the square $[0,1]^{2}$, can possess two limit cycles, an attracting one surrounded by a repelling one. This number may not be the maximum. However the result already shows the complexity of configurations that our apparently simple system can exhibit. Moreover we show how the two limit cycles can reduce to one, either repelling or attracting, through, respectively, a Hopf or a saddle-connection bifurcation, or can disappear through a collision (then the bifurcating cycle is interiorly attracting and externally repelling). In the latter case the vertex $(0,1)$ becomes the only attracting set of the system. Finally 


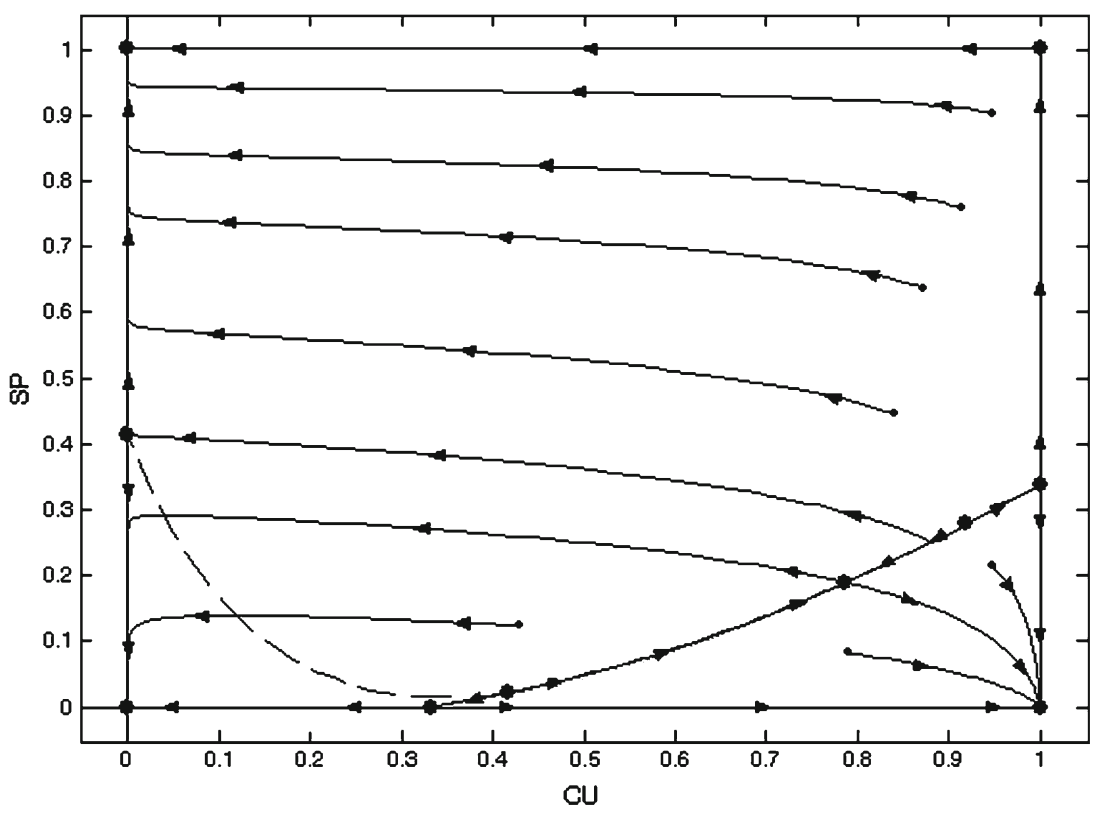

Fig. 2 Three interior fixed points. Parameters: $\lambda=11.45, \mu=36, v=0.25, \psi=0.25, \varphi=210, \omega=$ $10.0833, \theta=8, \tau=10, \sigma=0.5, \zeta=20.50$

we provide necessary and sufficient conditions for $(0,1)$ to attract all the trajectories lying in $(0,1)^{2}$.

We start with the following

Proposition 3 If system (2) exhibits some limit cycles in $(0,1)^{2}$, they all surround the same interior fixed point.

Proof It is well-known that the sum of the indexes of the fixed points surrounded by a limit cycle must be $+1^{3}$. As we have shown, if there are two non-degenerate interior fixed points, one of them is a saddle. Vice-versa, in the case the interior fixed points are three (accounting for multiplicity), Proposition 2 states that one of them (a repellor) cannot lie in the interior of a cycle. This proves the present Proposition.

Theorem 4 System (2) exhibits, for suitable values of the parameters, two limit cycles in $(0,1)^{2}$, an attracting one surrounded by a repelling one.

Proof We outline the main arguments of the proof, referring, for more technical details, to Antoci et al. (2011).

Let us first consider a system $\Sigma_{0}$ satisfying, besides (3), the following conditions:

1. $G_{0}(0,0)>0>G_{0}(1,1)$

2. $F_{0}(1,1)>0>F_{0}(1,0)$

\footnotetext{
3 For a non-degenerate fixed point the index is +1 if it is not a saddle, -1 if it is a saddle, while a saddle-node has index 0 etc. (see, e.g., Lefschetz 1977).
} 
3. There exists a fixed point $P_{0}=\left(x^{*}, 1\right), 0<x^{*}<1$, such that $F_{0}\left(x^{*}, 1\right)=0<$ $G_{0}\left(x^{*}, 1\right)$

4. There exists a fixed point $Q_{0}=\left(1, y^{*}\right), 0<y^{*}<1$, such that $F_{0}\left(1, y^{*}\right)=$ $G_{0}\left(1, y^{*}\right)=\frac{\partial G_{0}}{\partial y}\left(1, y^{*}\right)=0$

Conditions (1)-(4), in addition to the previous ones, are easily seen to imply:

- $\frac{\partial F_{0}}{\partial x}>0$ for $y \in\left[y^{*}, 1\right]$

- $\frac{\partial G_{0}}{\partial x}<0$ along $\left\{G_{0}(x, y)=0\right\} \cap[0,1]^{2}$

- $\frac{\partial G_{0}}{\partial y}>0$ along $\left\{G_{0}(x, y)=0\right\} \cap[0,1] \times\left[0, y^{*}\right)$

- $\frac{\partial G_{0}}{\partial y}<0$ along $\left\{G_{0}(x, y)=0\right\} \cap[0,1] \times\left(y^{*}, 1\right]$

It follows that $\Sigma_{0}$ has exactly six fixed points in $[0,1]^{2}$, all lying on the boundary: the attractor $(0,1)$, the saddles $(0,0),(1,0),(1,1),\left(x^{*}, 1\right)$ and the degenerate fixed point $Q_{0}=\left(1, y^{*}\right)$, whose Jacobian matrix has the form $J=\left(\begin{array}{ll}0 & 0 \\ -c & 0\end{array}\right)$ with $c>0$. Actually it can be checked that $Q_{0}$ possesses an elliptic sector and a parabolic sector lying in $(0,1)^{2}$, plus a hyperbolic sector lying outside the square ${ }^{4}$

Let us now perturb $\Sigma_{0}$ into $\Sigma$, by replacing $F_{0}$ and $G_{0}$ with:

$$
\begin{aligned}
& F(x, y)=F_{0}(x, y)+\alpha \varepsilon^{2} \\
& G(x, y)=G_{0}(x, y)-\varepsilon
\end{aligned}
$$

where $\varepsilon>0$ is arbitrarily small and $\alpha$ is suitably chosen. As a consequence, the fixed points of $\Sigma$ are the four vertices of $[0,1]^{2}$ and a point $P$ on $x=1$ near $P_{0}$ with the above characteristics, plus, in the place of $Q_{0}$, a point $Q$ in $(0,1)^{2}$, which, generically, is either an attractor or a repellor.

Moreover it can be proven (see Antoci et al. 2011) that, if $F$ is multiplied by a suitable $h>0$, then:

$$
\operatorname{trace} J(Q)=\beta \varepsilon^{2}+o\left(\varepsilon^{2}\right)
$$

Suppose, now, $Q$ is an attractor for $\Sigma$. Hence, since $\Sigma$ has no repelling fixed point in $[0,1]^{2}$, it follows from Poincaré-Bendixson Theorem (see Hirsch and Smale 1974) that there must exist a repelling limit cycle surrounding $Q$.

It can be checked that this cycle is not originated by a Hopf bifurcation. In fact, in such a case, its diameter should be, because of (5), of the order $\varepsilon^{2}$. But one can show (see Antoci et al. 2011) the existence of a winding inward trajectory starting at a point whose distance from $Q$ is $q \varepsilon$, with $q>0$ independent of $\varepsilon$. Hence this

4

- An elliptic sector of $P$ (see, e.g., Andronov et al. 1973) is constituted by trajectories having $P$ both as $\alpha$ and $\omega$ limit-set;

- A parabolic sector of $P$ is constituted by trajectories having $P$ either as $\alpha$ or as $\omega$ limit-set;

- A hyperbolic sector of $P$ is constituted by trajectories having $P$ neither as $\alpha$ nor as $\omega$ limit-set. 


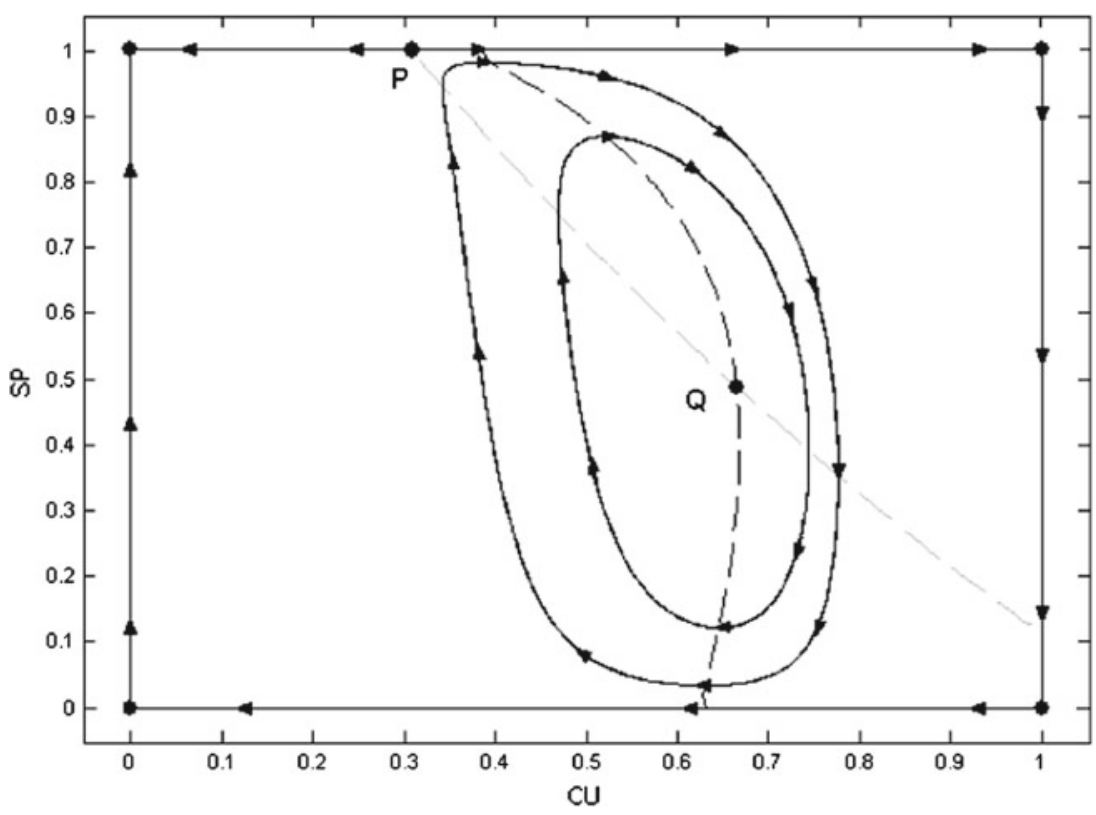

Fig. 3 Two limit cycles surrounding the only interior (repelling) fixed point $Q$. Parameters: $\lambda=520, \mu=$ $20, v=340, \psi=0.9, \varphi=7.5, \omega=96, \theta=140, \tau=94.5, \sigma=0.7, \zeta=93$

trajectory is contained in the region bounded by the repelling cycle and, consequently, the latter cannot be originated by a Hopf bifurcation. Therefore the Hopf bifurcation at $Q$, which generically takes place when $\alpha$ crosses a suitable value $\bar{\alpha}$, is supercritical, i.e. an attracting limit cycle arises around the fixed point $Q$ when it becomes a repellor. Thus, in this case, two (at least) limit cycles exist, an attracting one surrounded by a repelling one.

This concludes the proof.

Figure 3 shows the case illustrated in the Theorem: there is only one interior fixed point, a repellor, surrounded by two limit cycles, respectively attracting and repelling. Now we want to show how the phase picture of system (2) can evolve, through bifurcations, starting from the one described in the above Theorem: that is, from two limit cycles to one (repelling or attracting) to no one. In the latter case we will see that $(0,1)$ becomes an attractor for all the trajectories lying in $(0,1)^{2}$ (except the one at the repelling fixed point), which can be considered a desirable outcome of the model.

Let us summarize the situation described in the Theorem, when two limit cycles appear. The following conditions hold:

- $\frac{\partial F}{\partial x}>0$ along $\{F=0\} \cap[0,1]^{2}$ and $\frac{\partial G}{\partial x}<0$ along $\{G=0\} \cap[0,1]^{2}$.

- $F=0$ intersects the boundary of $[0,1]^{2}$ at the two (open) edges $x=1$ and $y=1$, while $G=0$ intersects the boundary of $[0,1]^{2}$ at the two (open) edges $y=0$ and $y=1$.

- At the point $P=(\bar{x}, 1)$, where $F(\bar{x}, 1)=0, G(\bar{x}, 1)>0$. 


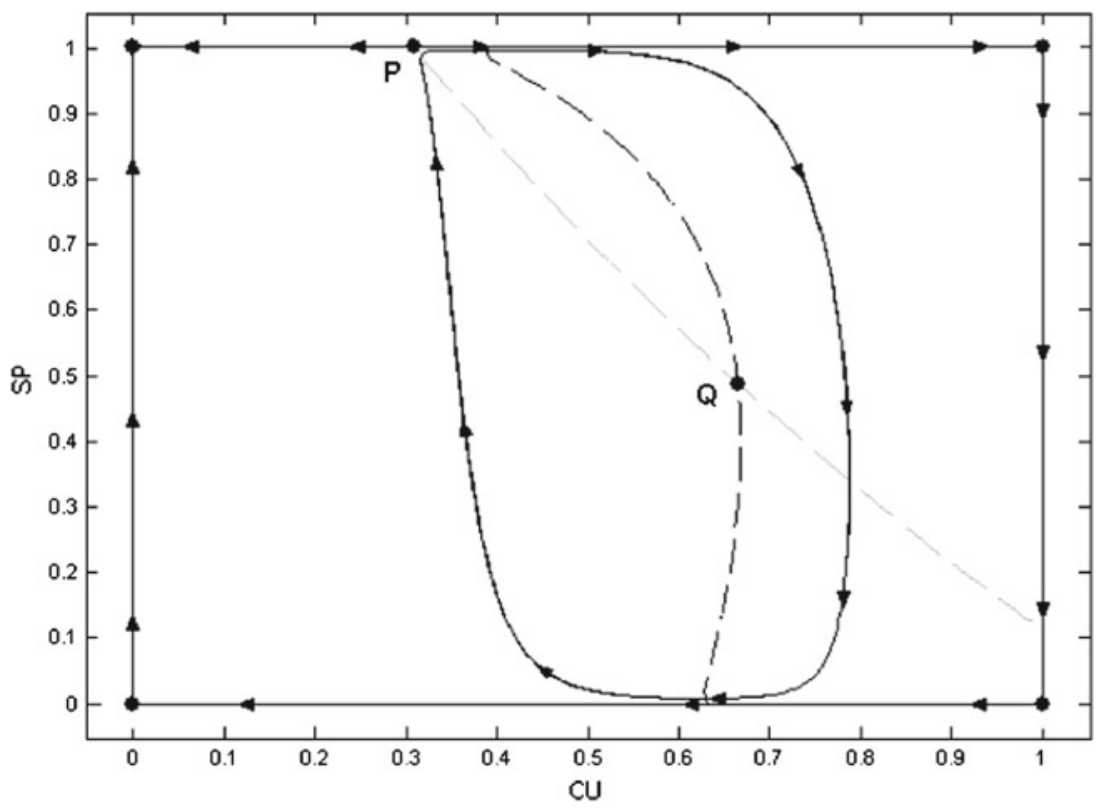

Fig. 4 One repelling limit cycle surrounding the only interior (attracting) fixed point $Q$. Parameters: $\lambda=520, \mu=20, v=340, \psi=0.663, \varphi=5.5252, \omega=70.7232, \theta=140, \tau=69.6182, \sigma=$ $0.7, \zeta=68.5131$

- Consequently there is only one interior fixed point $Q=(\widetilde{x}, \widetilde{y})$ and we assume $\frac{\partial G}{\partial y}(\widetilde{x}, \widetilde{y})<0$.

It follows that the fixed points of the system in $[0,1]^{2}$ are six: the attractor $(0,1)$, the saddles $(0,0),(1,0),(1,1),(\bar{x}, 1)$ and the interior fixed point $Q=(\widetilde{x}, \widetilde{y})$ which can be either an attractor or a repellor (observe that $\bar{x}<\tilde{x}$ ). In fact, multiplying $F$ by a suitable $h>0$, we can make $\operatorname{trace} J(Q)>0$, so that $Q$ is a repellor. In such a case there may exist, as we have seen, two limit cycles surrounding $Q$. Suppose we have precisely this phase picture. Now, if we multiply $F$ by a positive $h<1$, the attracting limit cycle shrinks, until it disappears when, at $h=\bar{h}, \operatorname{trace} J(Q)=0$. For smaller values of $h$ the system exhibits only one repelling limit cycle, surrounding $Q$ (see Fig. 4). This is the effect of a supercritical Hopf bifurcation. Let us see, instead, how only the attracting limit cycle can remain. By the usual notations, let us modify the coefficients $\zeta$ and $\omega$ of $F$, leaving all the others unvaried. Precisely, we replace $\zeta$ and $\omega$ by $\zeta^{\prime}=\zeta+\rho(\kappa)$ and $\omega^{\prime}=\omega+\rho(\kappa) \widetilde{x}, \rho(\kappa)>0$. At the same time we multiply $G$ by some $k>1$ in such a way that $\operatorname{trace} J(Q)$ remains positive but does not increase (or suitably decreases). Then, when $\rho(\kappa)$, starting from 0 , increases, the fixed point $P=(\bar{x}, 1)$ moves rightward, while the repelling limit cycle expands vertically, until, for a suitable pair $(\bar{\rho}(\bar{\kappa}), \bar{k}), F(\bar{x}, 1)=G(\bar{x}, 1)=0$. At this stage $P$ is a saddle-node and the repelling limit cycle has become a loop through $P$ (i.e. a saddle-connection). For higher values of $\rho(\kappa)$ there is only one attracting limit cycle surrounding $Q$, while $P$ has become a repellor and there exists a new interior fixed point, a saddle $S=(\widehat{x}, \widehat{y})$, 


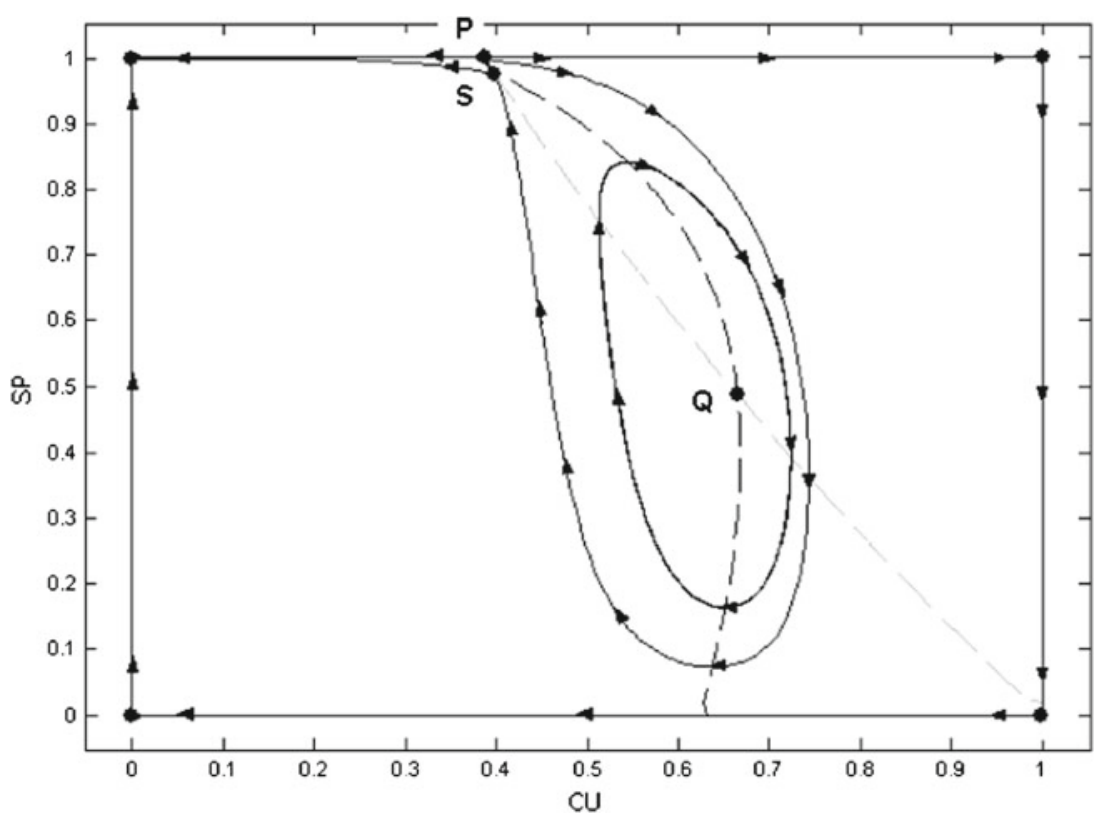

Fig. 5 One attracting limit cycle. Parameters: $\lambda=676, \mu=26, v=442, \psi=0.9, \varphi=7.5, \omega=$ 101.9827, $\theta=182, \tau=94.5, \sigma=0.7, \zeta=102$

with $\widehat{x}<\tilde{x}$ and $\hat{y}>\tilde{y}$. The basin of the attracting cycle is bounded, precisely, by the stable manifold of $S$, whose arcs originate in $P$ (see Fig. 5). We observe, however, that, by just letting the curve $F=0$ approach the curve $G=0$ on the edge $y=1$ of the square, without adjusting at the same time $\operatorname{trace} J(Q)$, the resulting phase picture might have been different: that is, when the boundary fixed point, say $\widetilde{P}$, becomes a repellor and an interior saddle $S$ appears, the two limit cycles may persist (see Fig. 6).

Finally we show that both limit cycles can disappear through a collision. In fact, let us start again from the two limit cycles configuration. Then, by multiplying $F$ by some $h>1$, trace $J(Q)$ increases, while the attracting limit cycle around $Q$ expands and the repelling one shrinks, until, at a suitable value $\overline{\bar{h}}$, they coincide (i.e. collide). The resulting compound limit cycle is interiorly attracting and externally repelling (see Fig. 7). Then, for $h>\overline{\bar{h}}$, no cycle exists and all the trajectories starting in $(0,1)^{2}$ (except, of course, the one at $Q$ ) converge to the virtuous point $(0,1)$.

From the analysis contained in this section it emerges that the basin of attraction of the desirable equilibrium $(x, y)=(0,1)$ may have a rather complex morphology, even when $(0,1)$ is the unique attractive fixed point, as it is shown in Fig. 3, where the repelling cycle separates the basin of attraction of the attractive one from that of the vertex $(0,1)$.

The path-dependence of evolutionary dynamics could be viewed as a shortcoming of the proposed fund rising mechanism. Nevertheless, we can give necessary and sufficient conditions for the global attractiveness of $(0,1)$ relatively to the open square $(0,1)^{2}$, namely: 


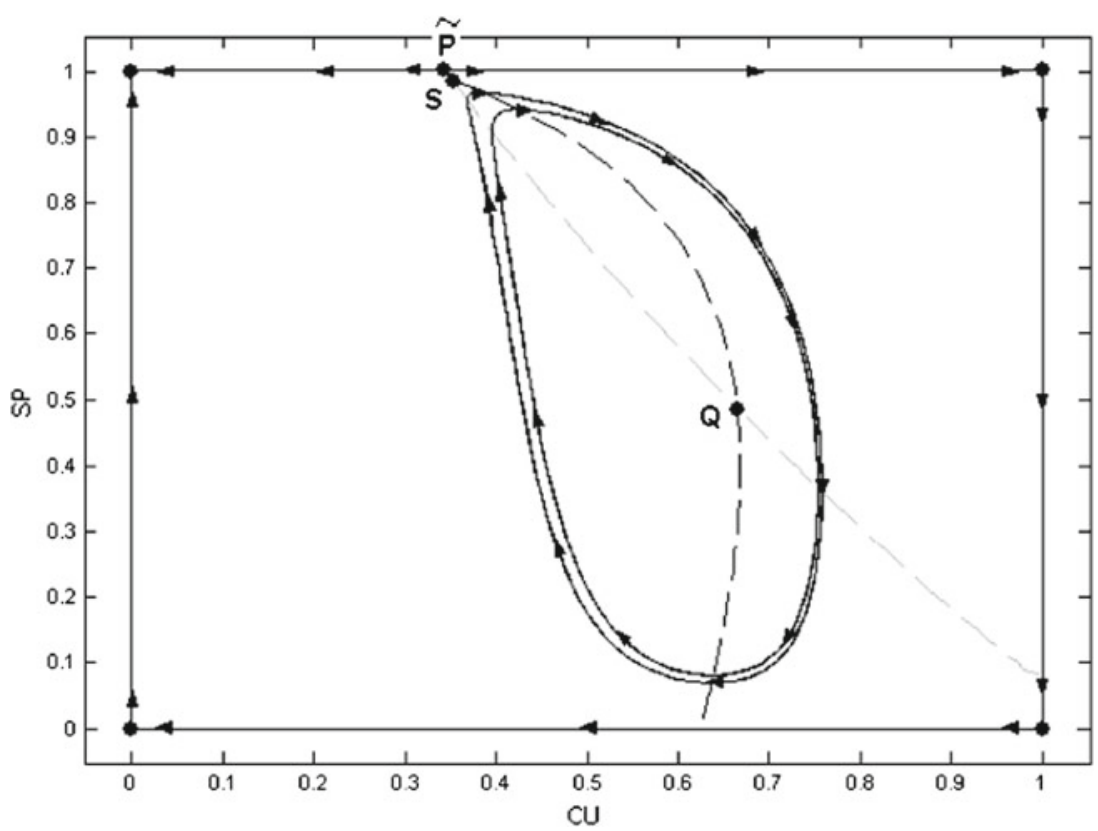

Fig. 6 Two limit cycles with two interior fixed points. Parameters: $\lambda=508.0347, \mu=38, v=340, \psi=$ $0.9, \varphi=7.5, \omega=98.3266, \theta=140, \tau=94.5, \sigma=0.7, \zeta=96.5$

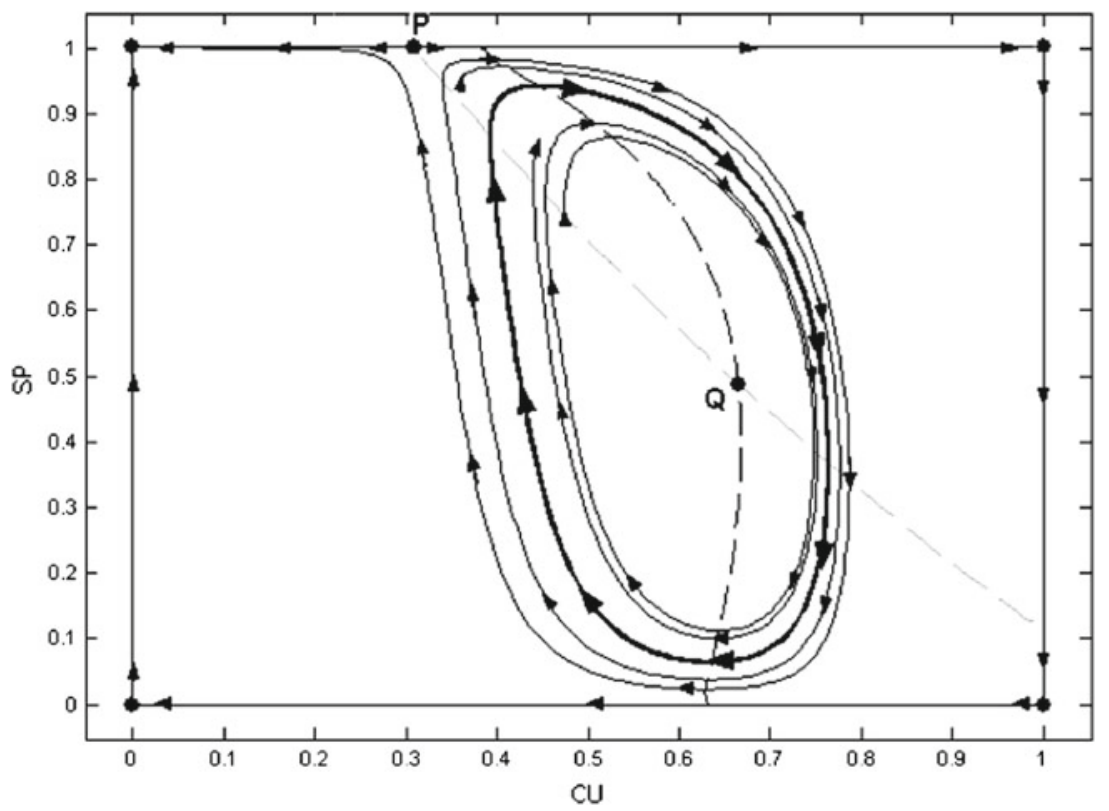

Fig. 7 One compound limit cycle (internally attracting and externally repelling). Parameters: $\lambda=$ 520, $\mu=20, v=340, \psi=0.9483, \varphi=7.9024, \omega=101.1504, \theta=140, \tau=99.5699, \sigma=$ $0.7, \zeta=97.9894$ 
Proposition 5 The vertex $(0,1)$ attracts all the trajectories lying in the open square $(0,1)^{2}$ if and only if the following conditions hold:
1. $\sigma \lambda \geq \theta$;
2. $\omega \geq \zeta$;
3. there exists $h>0$ such that $h F(1,1)+G(1,1) \leq 0$;
4. $F(x, y)<0$ along $\{G(x, y)=0\} \cap(0,1)^{2}$

We omit the proof of the above Proposition, which can be drawn through straightforward steps.

Let us observe that the first three conditions guarantee that the vertices, in the order, $(0,0),(0,1)$ and $(1,1)$ are not attractors, while the fourth condition, together with the other three, guarantees that no interior fixed point exists and no other boundary fixed point, except $(0,1)$, attracts trajectories lying in $(0,1)^{2}$.

We can also give a sufficient condition, which allows an easier economical interpretation, for the vertex $(0,1)$ to attract all the trajectories lying in $(0,1)^{2}$. This condition is obtained observing that $x<0$ holds for every values of $x$ and $y$ in the open square $(0,1)^{2}$ if:

$$
\min \left\{(1-\alpha) p_{a}-(1-\beta) p_{b}, p_{a}-p_{b}+\sigma\left(\beta p_{b}-\alpha p_{a}\right)\right\} \geq \frac{\eta-\delta}{\rho}
$$

Under condition (6) all the trajectories in $(0,1)^{2}$ approach the edge $x=0$ of the square. So, if both (6) and $\sigma \lambda \geq \theta$ are satisfied:

$$
\sigma \lambda \geq \theta \quad \text { and } \min \left\{(1-\alpha) p_{a}-(1-\beta) p_{b}, \quad p_{a}-p_{b}+\sigma\left(\beta p_{b}-\alpha p_{a}\right)\right\} \geq \frac{\eta-\delta}{\rho}
$$

then the vertex $(0,1)$ attracts all the trajectories lying in the open square $(0,1)^{2}$. The condition (7) requires (ceteris paribus):

(1) a sufficiently large difference $p_{a}-p_{b}$ between the prices of the tickets A and $\mathrm{B}$ bought by the $\mathrm{CU}$;

(2) a sufficiently large difference $\beta-\alpha$ between the reimbursement rates of the tickets $\mathrm{B}$ and $\mathrm{A}$, respectively;

(3) a sufficiently large fixed amount of money $\lambda$ (i.e. the component of the reward $\lambda+\mu x-v y$ not depending on the shares $x$ and $y$ ) received, when $Q \geq Q^{*}$, by each service provider deciding to provide high quality services;

(4) a high enough sensitivity (measured by the parameter $\sigma$ ) of the probability $P\left(Q \geq Q^{*}\right)=\sigma(1-x)+(1-\sigma) y$ with respect to variations in $x$, the share of CU using private cars.

Notice that, in (7), the threshold value $\frac{\eta-\delta}{\rho}$ increases (ceteris paribus) if the difference $\eta-\delta$ increases, where $\eta$ (respectively $\delta$ ) measures the negative impact due to an increase in $x$ on CU adopting choice $(b)$ (respectively $(a)$ ). In fact, in a context characterized by a high value of $\eta-\delta$, the decision to use a private car has a strong selfenforcing nature that favours the emergence of undesirable outcomes characterized by a widespread use of private cars. 


\section{Conclusions}

In this paper we have analyzed the effects on economic agents' behavior generated by the introduction of a simple mechanism aimed at implementing and supporting environmental protection policies in urban areas. According to the proposed mechanism, service providers choosing to offer high quality services can obtain cost abatement; while CU have to pay for entering into urban areas, but can protect themselves against a low quality of life in these areas by a self-insurance device. The dynamics arising from the interaction between these categories of economic agents are modelled by a two-population evolutionary game according to which the population of CU strategically interacts with that of service providers. In its turn the PM can achieve the goal of improving the CU quality of life at a low cost, since the costs born by CU compensate, at least partially, the financial aids to virtuous services providers and so do not imply any worsening of the public budget. However we did not introduce any explicit budget constraint, whose possible satisfaction can affect the dynamics of the replication system, due, in particular, to the value of the parameter $\rho$, expressing the citizen non-willingness to pay for urban services (see Sect. 2). Anyway for the always attracting equilibrium $(0,1)$ the budget constraint reduces to $p_{b} \geq \lambda-v$.

From the analysis of the model it emerges that the desirable equilibrium $(x, y)=$ $(0,1)$, where all CU choose an environment-preserving transport mode and all services agencies provide high quality services, is always a local attractor under evolutionary dynamics. However, the basin of attraction of such an equilibrium may have a rather complex morphology. In fact, in Sects. 3 and 4 we show that other attracting fixed points can coexist with $(0,1)$ and, furthermore, that two limit cycles may arise, an attracting one surrounded by a repelling one. The latter separates the basin of attraction of the former from the basin of attraction of the virtuous vertex $(0,1)$. Therefore, evolutionary dynamics is strongly path-dependent. This feature of dynamics may be viewed as a shortcoming of the proposed mechanism. Nevertheless we have given a sufficient condition (see (7)) assuring the global attractiveness of the equilibrium $(0,1)$.

The present analysis could be extended in several directions. In particular, it would be interesting to analyze the dynamics arising in an optimal control framework in which the PM aims at maximizing its own objective function by choosing the values of the control variables $p_{a}, p_{b}, \alpha$ and $\beta$.

Acknowledgements The authors wish to thank two referees of this journal, whose remarks and suggestions have allowed a substantial improvement of the work. The research of Angelo Antoci has been supported by a grant of the Fondazione Banco di Sardegna and that of Marcello Galeotti by a grant of the Istituto Nazionale di Alta Matematica Francesco Severi.

\section{References}

Andronov, A. A., et al. (1973). Qualitative theory of second-order dynamic systems. Jerusalem: John Wiley.

Antoci, A., \& Sodini, M. (2009). Indeterminacy, bifurcations and chaos in an overlapping generations model with negative environmental externalities. Chaos, Solitons and Fractals, 42, 1439-1450. 
Antoci, A., Borghesi, S., \& Russu, P. (2005). Environmental defensive expenditures, expectations and growth. Population and Environment, 27, 227-244.

Antoci, A., Galeotti, M., \& Russu, P. (2007a). Undesirable economic growth via economic agents' self-protection against environmental degradation. Journal of The Franklin Institute, 344, 377-390.

Antoci, A., Galeotti, M., \& Geronazzo, L. (2007b). Visitor and firm taxes versus environmental options in a dynamical context. Journal of Applied Mathematics, 2007(97540), 1-15.

Antoci, A., Marletto, G., Russu, P., \& Sanna, S. (2009a). I social policy bonds 'modificati' come strumento di regolazione del trasporto urbano: Una simulazione concettuale. In G. Marletto \& E. Musso (Eds.), Trasporti, ambiente e territorio. La ricerca di un nuovo equilibrio. Milan: Franco Angeli.

Antoci, A., Dei, R., \& Galeotti, M. (2009b). Financing the adoption of environment preserving technologies via innovative financial instruments: An evolutionary game approach. Nonlinear Analysis Series A: Theory, Methods \& Applications, 71(12), 952-959.

Antoci, A., Borghesi, S., \& Galeotti, M. (2009c). Environmental options and technological innovation: an evolutionary game model. Nota di lavoro FEEM n. 2009.090, Milan.

Antoci, A., Sodini, M., \& Naimzada, A. (2010). Bifurcations and chaotic attractors in an overlapping generations model with negative environmental externalities. In G. Bischi, C. Chiarella, \& L. Gardini (Eds.), Nonlinear dynamics in economics, finance and social sciences. Amsterdam: Springer Verlag.

Antoci, A., Galeotti, M., \& Radi, D. (2011). Financial tools for the abatement of traffic congestion: A dynamical analysis. DiMaD Working Papers 2011-01, Department of Mathematics for Decisions, University of Florence.

Bischi, G.-I., \& Radi, D. (2011). An extension of the Antoci-Dei-Galeotti evolutionary model for environment protection through financial instruments. Nonlinear Analysis: Real World Applications. doi:10.1016/j.nonrwa.2011.07.046

Bonatti, L., \& Campiglio, E. (2009). Mobility systems and economic growth: A theoretical analysis of the long-term effects of alternative transportation policies. Discussion Papers 7-2009, Department of Economics, University of Trento, Trento.

Costanza, R., \& Perrings, C. (1990). A flexible assurance bonding system for improved environmental management. Ecological Economics, 2, 57-75.

Gwilliam, K. (2008). A review of issues in transit economics. Research in Transportation Economics, 23, 4-22.

Hirsch, M. W., \& Smale, S. (1974). Differential equations, dynamical systems, and linear algebra. Orlando, FL: Academic Press.

Horesh, R. (2000). Injecting incentives into the solution of social and environmental problems: Social policy bonds. New York, NY: University Press.

Horesh, R. (2000). Injecting incentives into the solution of social problems. Social policy bonds. Economic Affairs, 20, 39-42.

Horesh, R. (2002). Better than Kyoto: Climate stability bonds. Economic Affairs, 22, 48-52.

Horesh, R. (2002b). Environmental policy bonds: Injecting market incentives into the achievement of society's environmental goals. OECD Paper, Paris.

Hueting, R. (1980). New scarcity and economic growth. More welfare through less production?. Amsterdam: North Holland.

Hull, J. (2005). Options, futures and other derivatives. Upper Saddle River, NJ: Prentice Hall.

Lefschetz, S. (1977). Differential equations: Geometric theory. New York, NY: Dover Publications.

Perrings, C. (1989). Environmental bonds and environmental research in innovative activities. Ecological Economics, 1, 95-110.

Torsello, L., \& Vercelli, A. (1998). Environmental bonds: A critical assessment. In G. Chichilnisky, G. Heal, \& A. Vercelli (Eds.), Sustainability: Dynamics and uncertainty. Amsterdam: Martin Kluwer.

Weibull, J. W. (1995). Evolutionary game theory. Cambridge, MA: MIT Press. 